\title{
Communication importante: Rappel à titre préventif de lots de Petinimid
}

\section{Petinimid 250 mg, 100 capsules de gélatine molle, numéro d'autorisation 29934}

En accord avec Swissmedic, axapharm ag rappelle le lot OA202A du médicament Petinimid 250 mg, 100 capsules de gélatine molle, à titre préventif jusqu'au niveau du commerce de détail.

Le rappel concerne uniquement le lot suivant de Petinimid:

\begin{tabular}{lll}
\hline Numéro de lot & Date d'expiration & $\begin{array}{l}\text { Période de } \\
\text { livraison }\end{array}$ \\
\hline OA202A & $\mathbf{1 2 / 2 0 2 4}$ & $13.8 .2020-$ \\
& & 12.2 .2021 \\
\hline
\end{tabular}

La raison du rappel est la fuite possible de capsules individuelles à l'intérieur du blister.
Nous vous prions de contrôler vos stocks et de séparer tous les emballages du lot mentionné ci-dessus du reste de votre stock et de ne plus livrer ou remettre au client.

Veuillez retourner immédiatement tous les emballages concernés de votre stock via le canal de distribution inverse. Si vous avez commandé des emballages auprès d'un grossiste, veuillez retourner les emballages en question à ce grossiste. Le paiement est également effectué par l'intermédiaire du grossiste. Nous nous tenons à votre disposition en cas de questions. Dans ce cas, veuillez s'il vous plaît contacter: quality@axapharm.ch

Pour le signalement de tout effet indésirable (EI), Swissmedic encourage les personnes concernées à utiliser l'outil de déclaration d'effets indésirables développé à cet effet. L'utilisation de l'Electronic Vigilance System (ElViS) permet de faire la déclaration des EI par saisie directe ou par téléchargement de fichier XML. Vous pourrez trouver toutes les informations nécessaires sous www.swissmedic.ch $\rightarrow$ «Signaler les risques".

Nous nous excusons pour les désagréments engendrés par le rappel des lots et vous remercions par avance de votre support.

axapharm ag

Zugerstrasse 32

CH-6340 Baar

Tél. 0417668383

info@axapharm.ch 